\title{
Bang-bang refocusing of a qubit exposed to telegraph noise
}

\author{
Henryk Gutmann and Frank K. Wilhelm \\ Sektion Physik and CeNS, Ludwig-Maximilians-Universität, 80333 München, Germany \\ William M. Kaminsky \\ Department of Physics, Massachusetts Institute of Technology, Cambridge, MA 02139 \\ Seth Lloyd \\ Department of Mechanical Engineering, \\ Massachusetts Institute of Technology, Cambridge, MA 02139
}




\begin{abstract}
Solid state qubits promise the great advantage of being naturally scalable to large quantum computer architectures, but they also possess the significant disadvantage of being intrinsically exposed to many sources of noise in the macroscopic solid-state environment. With suitably chosen systems such as superconductors, many of sources of noise can be suppressed. However, imprecision in nanofabrication will inevitably induce defects and disorder, such as charged impurities in the device material or substrate. Such defects generically produce telegraph noise and can hence be modelled as bistable fluctuators. We demonstrate the possibility of the active suppression of such telegraph noise by bang-bang control through an exhaustive study of a qubit coupled to a single bistable fluctuator. We use a stochastic Schrödinger equation, which is solved both numerically and analytically. The resulting dynamics can be visualized as diffusion of a spin vector on the Bloch sphere.

We find that bang-bang control suppresses the effect of a bistable fluctuator by a factor roughly equalling the ratio of the bang-bang period and the typical fluctuator period. Therefore, we show the bang-bang protocol works essentially as a high pass filter on the spectrum of such telegraph noise sources. This suggests how the influence of $1 / f$-noise ubiquitous to the solid state world could be reduced, as it is typically generated by an ensemble of bistable fluctuators. Finally, we develop random walk models that estimate the level of noise suppression resulting from imperfect bang-bang operations, such as those that cannot be treated as $\delta$-function impulses and those that have phase and axis errors.

PACS numbers: 03.65.Yz, 03.67.Lx, 05.40.-a
\end{abstract}




\section{INTRODUCTION}

In order to implement solid-state quantum information processing devices, the noise sources causing decoherence of their quantum states have to be carefully understood, controlled, and eliminated. This is a formidable task, as a solid-state environment generically couples a macroscopic number of degrees of freedom to any such device. Thus, a fundamental prerequisite for any design is that it must significantly decouple the quantum states used for computation from phonons and other quasiparticles in the underlying solid crystal. Examples of such designs are those employing discrete states in quantum dots [1] or superconductors with a gapped density of states [2, 3, 4, 5, 6].

Most research going beyond this fundamental prerequisite has concentrated on decoupling devices from external noise sources such as electromagnetic noise generated by control and measurement apparatus [7]. On the other hand, there inevitably are internal noise sources because the fabrication of gates, tunnel junctions, and other functional components creates defects in the underlying crystal. Prominent examples of such defects are background charges in charge-based devices or cricital current fluctuations in flux-based devices [8, 9]. A clear signature of such defects is telegraph noise in the case of a few defects or $1 / f$-noise in the case of a larger ensemble [10]. With the growing success in engineering the electromagnetic environment, these defects are becoming more and more the key limiting sources of decoherence.

Such defects do not fall in the large class of noise sources that can be approximated well as a bosonic bath, and this fact complicates analysis. Whereas it is realistic to treat a bosonic bath in the tractable near-equilibrium thermodynamic limit where fluctuations are purely Gaussian [11, 12, 13], localized noise sources with bounded spectra like the defects in which we are interested produce noise that is significantly non-Gaussian. Theories treating large ensembles of non-Gaussian noise sources have been presented [14, 15]. However, with the ongoing improvement in nanofabrication technology, it is realistic to consider the case where non-Gaussian noise sources are reduced down to only a single one or a few per device. This is the case we treat here, and thus the defects find a more realistic representation as a small set of bistable fluctuators [16] (henceforth abbreviated bfls). In principle, this approach can be extended to larger sets of bfls with a range of different mean switching times (e.g., an ensemble with an exponential distribution of switching times that produces $1 / f$-noise 
[17, 18, 19]).

This report is organized as follows. Section II presents the model of a single bfl in the semiclassical limit, where it acts as a source of telegraph noise. Section III introduces an idealized open loop quantum control technique, quantum bang-bang control [20, 21, 22], which is suitable for slowly fluctuating noise sources. Section IV explains how we simulated the qubit dynamics under the influence of noise with and without bang-bang control by integrating of the corresponding time-dependent Schrödinger equation. As a measure of the decoherence, we analyze the deviations of the qubit's trajectory on the Bloch sphere from that of the noiseless case. These deviations take the form of a random walk around the noiseless-case trajectory. We therefore analyze the suppression of these deviations by comparing the variances of these random walks with and without bang-bang control. Both numerical and analytical solutions (the latter in the long-time or "diffusion" limit) are presented. Comparison of the numerical simulations to the analytical solutions shows excellent agreement. We then analyze how these results change when practical limitations are considered such as the fact that a bang-bang pulse cannot be an ideal $\delta$-function impulse and the fact that the duration or polarization axis of the pulse may suffer from random fluctuations. We show at the end of Section IV.B that within large margins bang-bang suppression of the bfl noise is not inhibited by having a finite, rather than infinitesimal, pulse length. However, in Section V, we do find that duration and polarization axis errors in the bang-bang pulses can significantly affect the suppression of bfl noise. We present a point of optimum performance. Section VI concludes with remarks on several recent publications concerning the suppression of telegraph or $1 / f$-noise.

\section{MODEL OF THE BISTABLE FLUCTUATOR IN ITS SEMICLASSICAL LIMIT}

We describe the bfl-noise influenced evolution of the qubit in its semiclassical limit by using a stochastic Schrödinger equation [23, 24] with the time-dependent effective Hamiltonian

$$
\begin{aligned}
H_{q}^{\mathrm{eff}}(t) & =H_{\mathrm{q}}+H_{\text {noise }}(t) \\
H_{\mathrm{q}} & =\hbar \epsilon_{\mathrm{q}} \hat{\sigma}_{\mathrm{z}}^{\mathrm{q}}+\hbar \Delta_{\mathrm{q}} \hat{\sigma}_{\mathrm{x}}^{\mathrm{q}} \\
H_{\text {noise }}(t) & =\hbar \alpha \hat{\sigma}_{\mathrm{z}}^{\mathrm{q}} \xi_{\mathrm{bfl}}(t)
\end{aligned}
$$


where $\epsilon_{\mathrm{q}}$ and $\Delta_{\mathrm{q}}$ define the free (noiseless) qubit dynamics. $\xi_{\mathrm{bfl}}(t)$ denotes a function randomly switching between \pm 1 (see Fig. 1), which represents a telegraph noise signal. The switching events follow a symmetrical Poisson process, i.e., the probabilities of the bfl switching from +1 to -1 or -1 to +1 are the same and equal in time. The Poisson process is characterized by the mean time separation $\tau_{\text {bf }}$ between two bfl flips. The coupling amplitude to the qubit in frequency units is $\alpha$. The relation of this Hamiltonian to a microscopic model is explained in the Appendix.

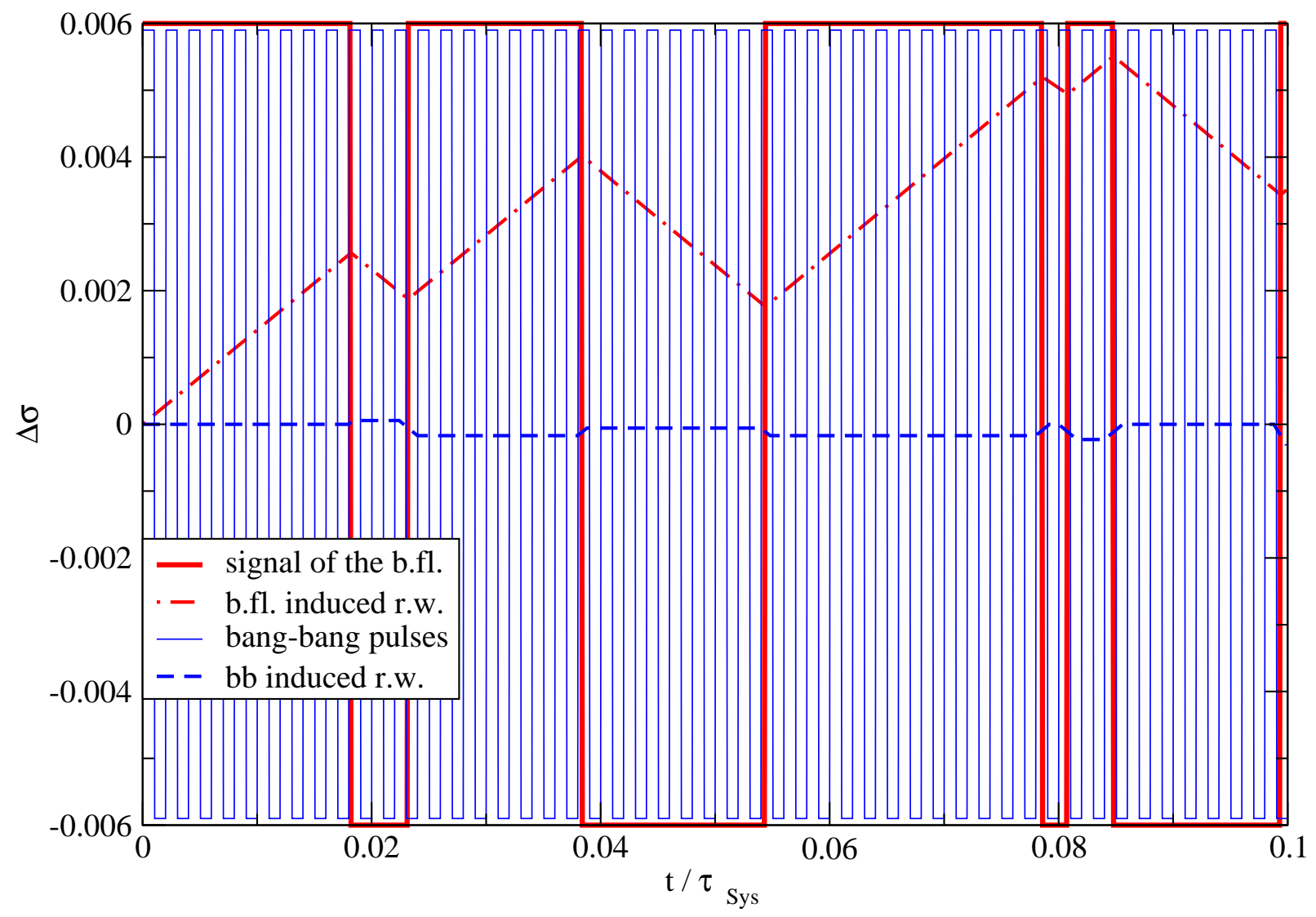

FIG. 1: Schematic plot of a typical Poisonian bfl noise signal and its resulting random walk behavior (in the limit of small deviations). The periodic fast switching step function represents a bang-bang pulse with a time scale ratio: $\tau_{\mathrm{bff}} / \tau_{\mathrm{bb}}=10$ and yields a quite smaller random walk step-length. $\tau_{\text {Sys }}=\frac{\pi}{\sqrt{\epsilon_{\mathrm{q}}^{2}+\Delta_{\mathrm{q}}^{2}}}$ denotes the evolution period of the qubit in the noiseless case.

Starting with an arbitrary initial state of the qubit, represented by some given point 
on the Bloch sphere, we can numerically integrate the corresponding stochastic differential equation and obtain the corresponding random walk on the Bloch sphere

$$
\vec{\sigma}(t)=\mathrm{T} \exp \left(-i / \hbar \int_{0}^{t} H_{\mathrm{q}}^{\mathrm{eff}}(s) d s\right) \vec{\sigma}(0)
$$

with $\mathrm{T}$ denoting the usual time-ordering operator.

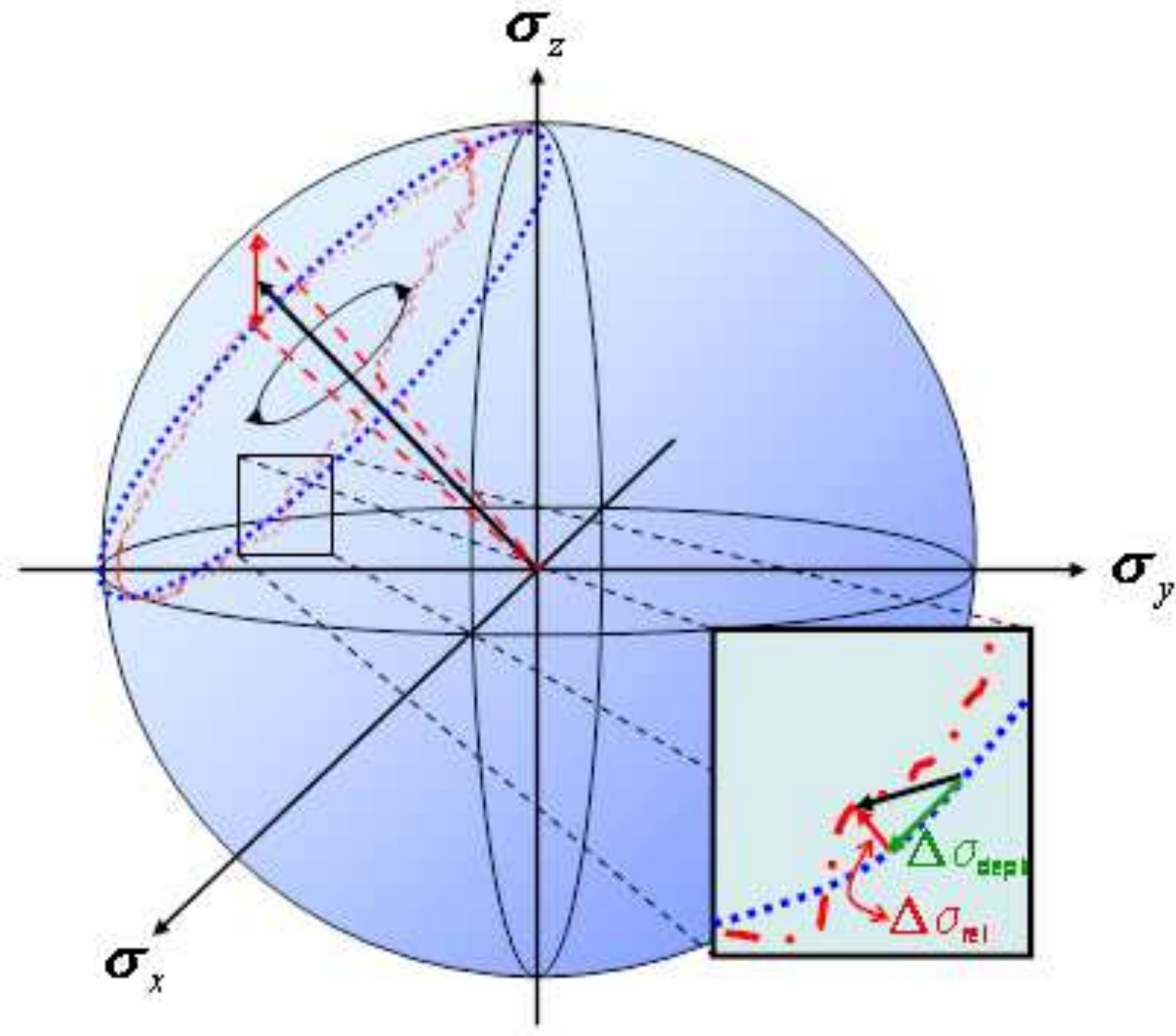

FIG. 2: Schematic plot of noisy qubit evolution generated by Poissonian telegraph noise. The resulting random walk (dot-dashed line) on the Bloch sphere is comprised both of deviations $\Delta \sigma_{\text {deph }}$ in parallel to the free precession trajectory (dotted line), which correspond to dephasing, and deviations $\Delta \sigma_{\text {rel }}$ perpendicular to it, which correspond to relaxation/excitation. 


\section{BANG-BANG CONTROL PROTOCOL}

We propose to reduce the influence of the bfl-noise by applying to the qubit a continuous train of $\pi$-pulses along the $\sigma_{\mathrm{x}}$-axis. This refocusing pulse scheme essentially corresponds to the standard quantum bang-bang procedure [20, 21, 22] or the Carr-Purcell-Gill-Meiboom echo technique from NMR [25]. For technical convenience, we consider the $\pi$-pulses to be of infinitesimal duration. This simplification is not crucial as will be detailed later in Section IV.B. The pulses are assumed to be separated by a constant time interval $\tau_{\mathrm{bb}}$. The mean separation $\tau_{\text {bf }}$ between two bfl flips is assumed to be much longer than $\tau_{\text {bb }}$. For theoretical convenience, we also assume that $\tau_{\mathrm{bff}}$ is shorter than the free precession period of the qubit. This too is not a crucial restriction. (It can always be overcome by changing to a co-precessing frame.)

Qualitatively, bang-bang control works as follows. Since $\tau_{\mathrm{bb}} \ll \tau_{\text {bfl }}$, it is usually the case that the bfl does not flip during the time between two bang-bang pulses that flip the qubit. In this way, the bang-bang pulses average out the influence of $H_{\text {noise }}(t)$. In fact, the refocusing scheme fully suppresses the $\sigma_{z}$-term of the static Hamiltonian (2) (compare Fig. (5); but this turns out to be no crucial obstacle to universal quantum computation as will be outlined later on. As one can visualize in Fig. 1, it is only when a bfl flip occurs during a bang-bang period that the net influence of the bfl felt by the qubit is nonzero, and the qubit thus suffers some random deviation from its trajectory in the noiseless case. Taken together, these random deviations constitute a random walk around the noiseless case trajectory. While this walk is actually continuous, it can be modelled as a discrete walk with steps that are randomly distributed in time, one step for each bfl flip (see e.g. [26]). The average step length is essentially the product of the noise coupling strength $\alpha$ and the mean time the bfl in its present state can influence the qubit. Without bang-bang control, this

mean influence time is $\tau_{\mathrm{bfl}}$, whereas with bang-bang control, it is reduced to $\tau_{\mathrm{bb}}$. Therefore, both with and without bang-bang control, the random walk has the same time distribution of steps, but with bang-bang control the step size can be significantly reduced roughly by a factor of the ratio of time scales $\tau_{\mathrm{bb}} / \tau_{\mathrm{bfl}}$. 


\section{RANDOM WALK ON THE BLOCH SPHERE}

Now we study this proposal quantitatively. We simulate these random walks both with and without bang-bang control by integrating both numerically and analytically the Schrödinger equation, Equ. (4), with the stochastic Hamiltonian of Equs. (1-3). As generic conditions for the qubit dynamics, we choose $\epsilon_{\mathrm{q}}=\Delta_{\mathrm{q}} \equiv \Omega_{0}$. Without loss of generality, we set the qubit's initial state to be spin-up along the $z$-axis. If the qubit-bfl coupling $\alpha$ were zero, then the qubit would simply precess freely on the Bloch sphere around the rotation axis $\hat{\sigma}_{\mathrm{x}}^{\mathrm{q}}+\hat{\sigma}_{\mathrm{z}}^{\mathrm{q}}$ (the dotted line in Fig. 2). Hence, we expect for a sufficiently small coupling $\left(\alpha \ll \Omega_{0}\right)$ only a slight deviation of the individual time evolution compared to the free evolution case (the dashed line in Fig. 2). For the coupling strength, we take $\alpha=0.1 \Omega_{0}$. All the following times and energies are given in units of the unperturbed system Hamiltonian, i.e., our time unit $\tau_{\text {Sys }}$ is given according the free precession time $\pi \tau_{\text {Sys }} / \sqrt{2}$, and our energy unit is given by $\Delta E=\sqrt{\epsilon_{\mathrm{q}}^{2}+\Delta_{\mathrm{q}}^{2}}=\sqrt{2} \Omega_{0}$. The time scale ratio is taken to be $\tau_{\mathrm{bfl}} / \tau_{\mathrm{bb}}=10$ if not denoted otherwise.

This approach accounts for the essential features of our specific situation: the long correlation time of the external noise, essentially $\tau_{\mathrm{bff}}$, its non-Gaussian statistics and its potentially large amplitude at low frequencies. These properties are crucial and are difficult, although not impossible, to take into account in standard master equation methods.

\section{A. Numerical simulations}

We have numerically integrated Equ. (41) and averaged the deviations of the random walk evolution from the unperturbed trajectories for times up to $100 \tau_{\text {Sys }}$ over $N=10^{3}$ realizations. Larger simulations have proven that convergence is already sufficient at this stage. We shall examine the root-mean-square (rms) deviations of this ensemble at given time points

$$
\Delta \vec{\sigma}_{\text {rms }}(t)=\sqrt{\frac{1}{N} \sum_{j=1}^{N}\left(\vec{\sigma}_{j}^{\mathrm{q}}(t)-\vec{\sigma}_{\text {noisy }, j}^{\mathrm{q}}(t)\right)^{2}}
$$

with and without bang-bang control. In other approaches, such as those based on master equations, one separates dephasing and relaxation. Both are contained here in Equ. (5). We shall point out notable differences between these two channels. The deviation as a function 


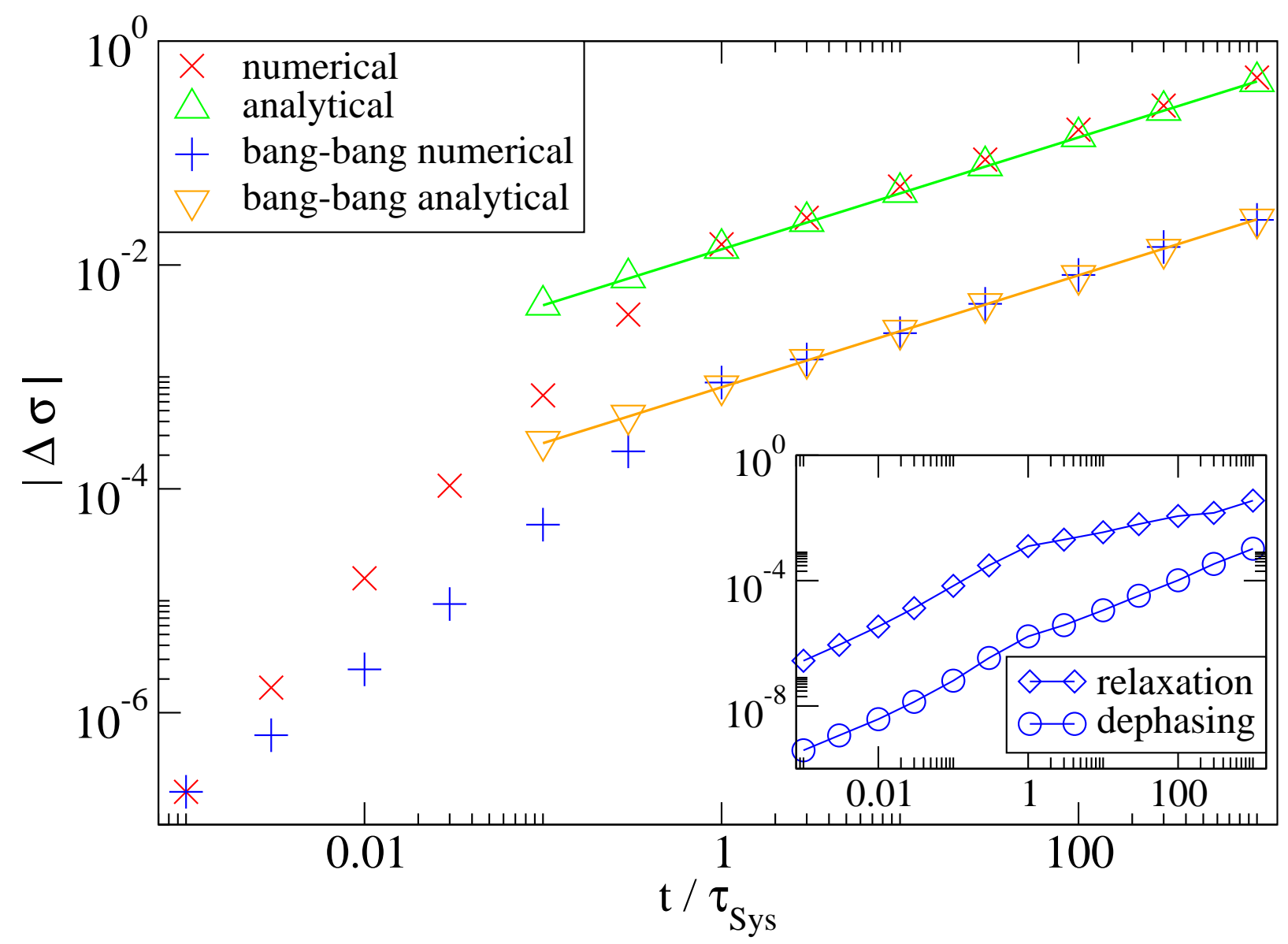

FIG. 3: Time evolution of the rms deviations for bfl-induced random walks with and without bang-bang control at a coupling constant $\alpha=0.1$ and a typical flipping time scale $\tau_{\text {bfl }}=10^{-2} \tau_{\text {Sys }}$. The separation between two bang-bang pulses is $\tau_{\mathrm{bb}}=10^{-3} \tau_{\text {Sys }}$. The straight lines are squareroot fits of the analytical derived random walk model variances (plotted as triangles). Inset: Components of the deviations from the free precession trajectory that are parallel to it (dephasing) and perpendicular to it (relaxation/excitation) with bang-bang control.

The total deviations on intermediate time scales are suppressed by a ratio on the order of 10. A detailed numerical analysis shows that without bang-bang suppression, the deviations parallel to the free precession trajectory (which correspond to dephasing) are of similar size to those perpendicular to free precession (which correspond to relaxation/excitation). In contrast, with bang-bang control, dephasing is almost totally absent as one can see in the inset of Fig. 3 .

The main double-logarithmic plot of Fig. 3 shows that on short time scales $\left(t \lesssim 0.1 \tau_{\text {Sys }}\right.$, 
which corresponds to $\lesssim 10$ random walk steps), deviations increase almost linearly in time. It is not until times on the order of $\tau_{\text {Sys }}$ that the noise-induced deviations start to behave as typical classical random walks, increasing as a square-root in time.

\section{B. Analytical random walk models}

We now develop analytical random walk models for our system. The random walk on the Bloch sphere is in general two-dimensional, consisting of both parallel and perpendicular deviations to the free evolution trajectory. Bang-bang control, as was seen in the above numerical results and as will be seen in the following analytical results, essentially reduces the random walk to one-dimension as only the perpendicular deviations remain significant. In the following, we restrict ourselves to the long-time (many random walk steps) regime.

We first calculate for both cases the probability distributions of the deviations after one bfl flip ("one-step deviations" in terms of the discrete random walk). The fluctuation of the period between $\tau_{\text {per }}^{ \pm}$leads to dephasing, which can be evaluated at $\alpha \ll \epsilon_{\mathrm{q}}, \Delta_{\mathrm{q}}$ to

$$
\Delta \vec{\sigma}_{\mathrm{deph}}^{\mathrm{bfl}}=2 \pi \cos \phi\left(\frac{1}{\tau_{\text {per }}^{ \pm}}-\frac{1}{\tau_{\text {per }}}\right) \tau_{\mathrm{bfl}} \simeq \pm 2 \frac{\Delta_{\mathrm{q}} \epsilon_{\mathrm{q}}}{\Delta_{\mathrm{q}}^{2}+\epsilon_{\mathrm{q}}^{2}} \alpha \tau_{\mathrm{bfl}}
$$

where the prefactor $\cos \phi=\frac{\Delta_{\mathrm{q}}}{\sqrt{\Delta_{\mathrm{q}}^{2}+\epsilon_{\mathrm{q}}^{2}}}$ takes the effective trajectory radius into account.

For the relaxation/excitation effect of the noise, one has to use the projection of the perturbation orthogonal to the free axis, using $\sin \eta=\frac{\alpha \Delta_{\mathrm{q}}}{\epsilon_{\mathrm{q}}^{2}+\Delta_{\mathrm{q}}^{2}}$. Furthermore this type of deviation also depends on the actual position of the spin on the Bloch sphere, e.g. there is no relaxation when the state is at one of the poles.

Averaging in rms-fashion over a full azimuthal cycle leads to a factor of $1 / \sqrt{2}$. Moreover, the impact of relaxation/excitation is scaled down by an additional factor of $\cos \phi=\frac{\Delta_{\mathrm{q}}}{\sqrt{\Delta_{\mathrm{q}}^{2}+\epsilon_{\mathrm{q}}^{2}}}$ corresponding to the projection of the Bloch vector onto the precession axis, which furthermore decreases the deviation angle. In total, using $\tau_{\text {per }}^{ \pm} \simeq \tau_{\text {per }}$ to first order in $\alpha$, we find

$$
\Delta \vec{\sigma}_{\text {rel }}^{\mathrm{bfl}}=2 \pi \cos \phi \sin \eta \frac{1}{\sqrt{2}} \cos \phi \frac{\tau_{\mathrm{bff}}}{\tau_{\mathrm{per}}^{ \pm}} \simeq \sqrt{2} \frac{\Delta_{\mathrm{q}}^{3}}{\left(\epsilon_{\mathrm{q}}^{2}+\Delta_{\mathrm{q}}^{2}\right)^{3 / 2}} \alpha \tau_{\mathrm{bff}} .
$$

Our root-mean-square measure of the impact of the noise, Equ. (5), does not handle these 


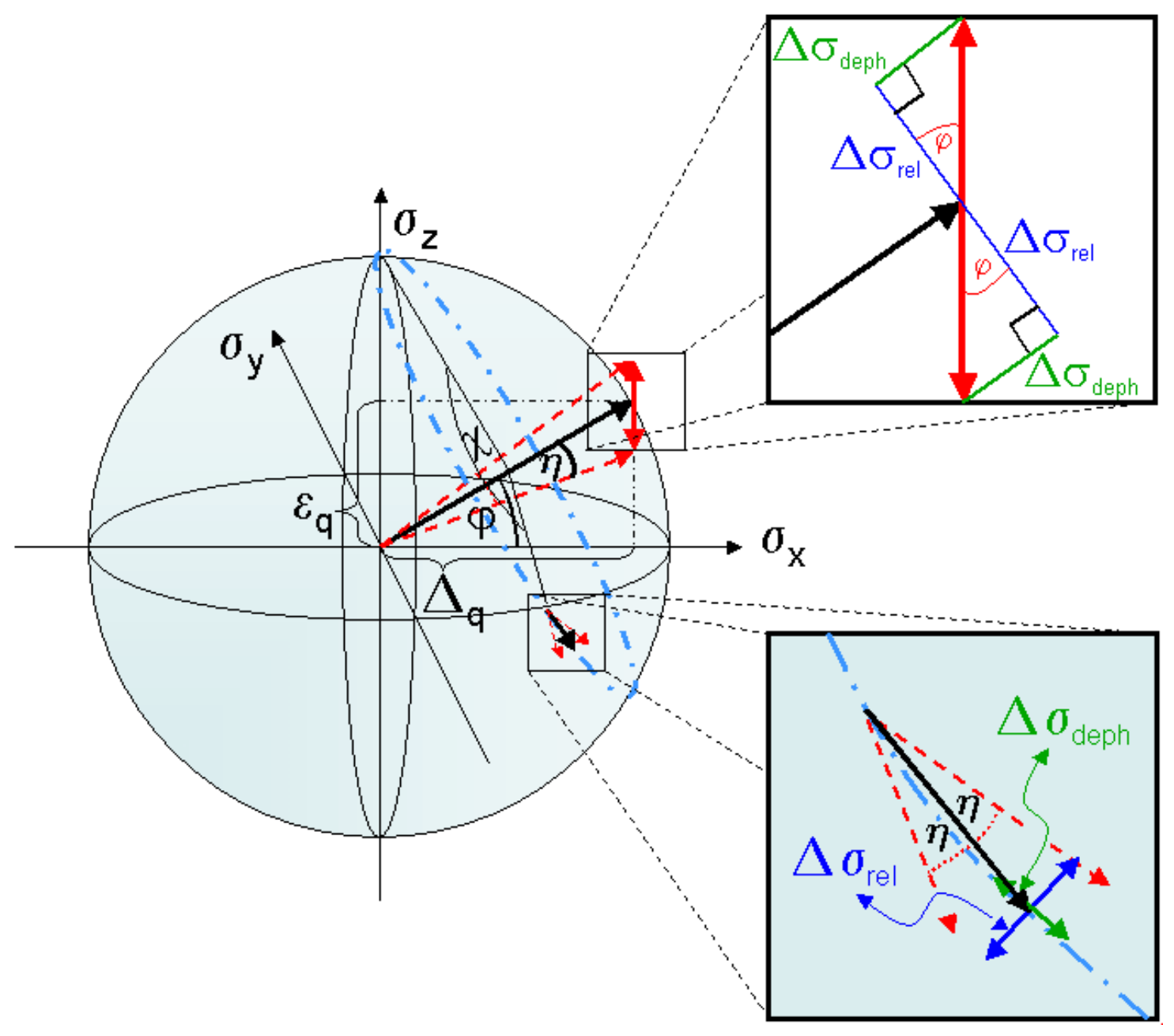

FIG. 4: Plot of a typical one-step deviation from the unperturbed qubit trajectory with generic values for $\epsilon_{\mathrm{q}}$ and $\Delta_{\mathrm{q}}$. The fractions of the bfl fluctuations in $\hat{\sigma}_{\mathrm{z}}$-direction have to be distinguished with respect to their effects on the qubit: those that yield dephasing deviations that are parallel to the free precession trajectory (proportional to $\sin \phi$ ) versus relaxation/excitation deviations that are perpendicular (proportional to $\sin \eta$ ). Both parts are additionally domineered by a factor of $\cos \phi$ due to the diminished radius of the trajectory starting from the initial state $\sigma_{z}=+1$. The impact of the relaxation/excitation generating part is furthermore depending on $\cos \phi$ as well as $\sin \chi$, the azimuth angle of the qubits present position.

two kinds of deviations separately, but rather adds them up to:

$$
\begin{aligned}
\Delta \vec{\sigma}_{\text {total }}^{\mathrm{bfl}}=\sqrt{\Delta \vec{\sigma}_{\mathrm{deph}}^{\mathrm{bfl}}{ }^{2}+\Delta \vec{\sigma}_{\mathrm{rel}}^{\mathrm{bfl}}}{ }^{2} & =\sqrt{4 \frac{\Delta_{\mathrm{q}}^{2} \epsilon_{\mathrm{q}}^{2}}{\left(\Delta_{\mathrm{q}}^{2}+\epsilon_{\mathrm{q}}^{2}\right)^{2}}+2 \frac{\Delta_{\mathrm{q}}^{6}}{\left(\epsilon_{\mathrm{q}}^{2}+\Delta_{\mathrm{q}}^{2}\right)^{3}}} \alpha \tau_{\mathrm{bfl}} \\
& =\frac{1}{\left(\Delta_{\mathrm{q}}^{2}+\epsilon_{\mathrm{q}}^{2}\right)^{3 / 2}} \sqrt{4\left(\Delta_{\mathrm{q}}^{2}+\epsilon_{\mathrm{q}}^{2}\right) \Delta_{\mathrm{q}}^{2} \epsilon_{\mathrm{q}}^{2}+2 \Delta_{\mathrm{q}}^{6}} \alpha \tau_{\mathrm{bfl}}
\end{aligned}
$$


Our rms treatment disregard the different types of decoherence, dephasing and relaxation/excitation, corresponding to phase and bit-flip errors respectively. This is no crucial drawback but merely lies in the nature of our generic situation. If needed, both components can be isolated.

The derivation of the maximal one-step deviation for the bang-bang controlled situation has to be handled differently. The deviation resulting from a bfl flip during a bang-bang pulse period is maximal if the step happens exactly at the moment of the second qubit spin-flip (i.e., in the middle of the bang-bang cycle). When this happens, the refocusing evolution has in its first half a drift, for example, to the "right" (compare to Fig. 5]) and in the last half an equal aberration.

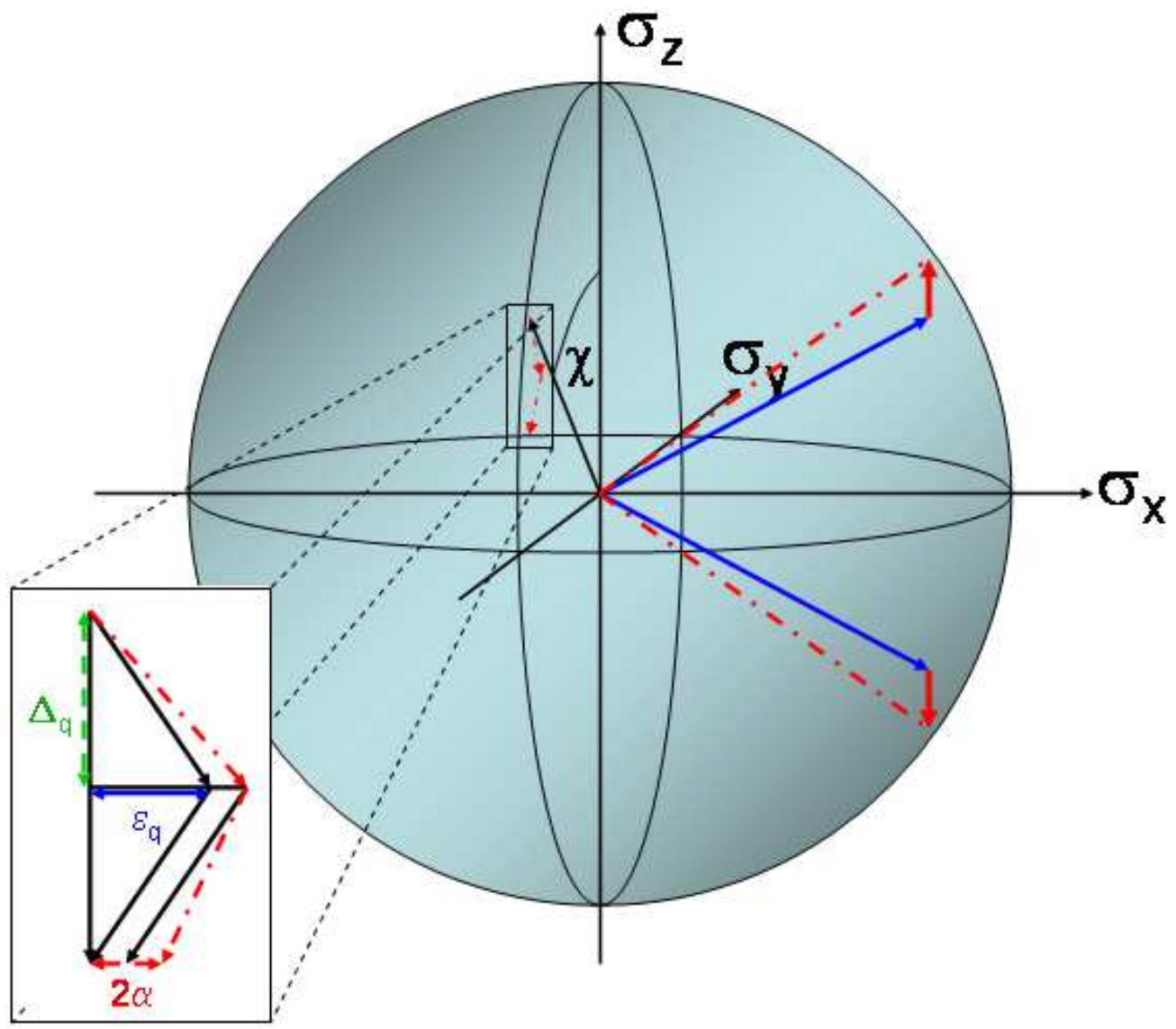

FIG. 5: Sketch of a maximal one-step deviation during a bang-bang modulated cycle, which appears if the bfl state flips precisely at the intermediate bang-bang pulse time. The dephasing part of deviation evidently averages out, while a relaxating aberrance arise proportional to the noise-coupling constant $\alpha$. 
The resulting one-step deviation appears to be on the order of $2 \alpha \tau_{\mathrm{bb}}$. However, this is scaled down by a factor of $1 / \sqrt{2}$, as the impact of the aberration in $x$-direction is proportional to a factor of $\sin \chi$, where $\chi$ denotes the longitudinal angle of the present spin position on the Bloch sphere (see Fig. 51). This is because the $\sigma_{z}$-component of the noisy evolution does not influence the qubit, if it is near the $\sigma_{z}= \pm 1$-state and its influence is suppressed correspondingly in between. As we are mainly interested in mean aberrations after many random walk steps, we simply average the maximal one-step deviation over one precession period in the usual rms manner to obtain

$$
\begin{aligned}
\left\langle\Delta \vec{\sigma}_{\max }^{\mathrm{bb}}\right\rangle & =\sqrt{\frac{1}{2 \pi} \int_{0}^{2 \pi} \sin ^{2} \chi 4 \alpha^{2} \tau_{\mathrm{bb}}^{2} d \chi} \\
& =\sqrt{2} \alpha \tau_{\mathrm{bb}} .
\end{aligned}
$$

Obviously, this variance only contributes to relaxation.

In the long time limit, we replace the fluctuating number of random walk steps for a given time $\Delta t$ of noisy evolution by its expectation value $N_{\mathrm{bfl}}=\Delta t / \tau_{\mathrm{bfl}}$. This allows us to use the number of random walk steps as time parameter [26]. This simplification does not introduce significant error, as the relative number variation for $\Delta t$ scales as $\frac{\sqrt{\Delta t / \tau_{\mathrm{bff}}}}{\Delta t / \tau_{\mathrm{bfl}}}=\sqrt{\tau_{\mathrm{bfl}} / \Delta t} \rightarrow 0$ in our preferred long-time limit. We encounter two different one-step-distributions, depending on whether the numeration of the step is an odd or even (corresponding to an "up" or "down" state of the bfl). For definiteness, we assume the bfl is initially in its "upper" state, which is of no influence on the long time limit as the memory to the initial state is already erased. The step-size distribution of the bfl model in our small deviation regime is given from Poisson statistics

$$
\Phi_{\text {odd } / \text { even }}^{\text {bfl }}(x)=\frac{e^{\mp x / \beta} \theta( \pm x)}{\beta}
$$

with $\beta=\frac{\sqrt{5}}{2} \alpha \tau_{\text {bfl }}$ the typical one-step deviation as calculated in Equ. 8, $\theta(x)$ denotes the Heaviside step function. We neglect the correlations between transverse and perpendicular deviations as we expect them to average out in the long-time limit.

For the bang-bang suppressed random walk, the flipping positions of the bfl-noise sign in the bang-bang time-slots are essentially randomly distributed as long as $\tau_{\text {bb }} \ll \tau_{\text {bfl }}$. That is why we find a constant step-size distribution between zero and a maximum divergence of $\gamma=\frac{2 \alpha \tau_{\mathrm{bb}}}{\sqrt{2}}$ (see Equ. 9) , namely

$$
\Phi_{\text {odd } / \text { even }}^{\mathrm{bb}}(x)=\frac{\theta( \pm x) \theta(\gamma \mp x)}{\gamma} .
$$


By means of these one-step probability distributions, we are able to calculate via the

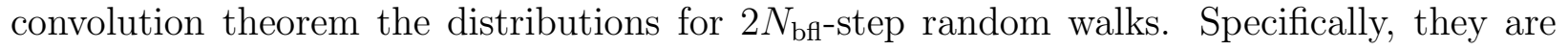
the inverse Fourier transforms of the $N_{\mathrm{bff}}$-fold products of the Fourier transforms of the two-step distribution [26]. For the case without bang-bang control, we find

$$
\begin{aligned}
\Phi_{2 \mathrm{~N}_{\mathrm{bf}}}^{\mathrm{bf}}(x) & =\mathscr{F}^{-1}\left[\left(\mathscr{F}\left[\Phi_{2}^{\mathrm{bff}}\right]\right)^{N_{\mathrm{bfl}}}\right] \\
& =\int_{-\pi}^{\pi} \frac{d k}{2 \pi \beta^{2 N_{\mathrm{bff}}}} e^{-i k x}\left(\frac{1}{1-2 \cos (k) e^{-1 / \beta}+e^{-2 / \beta}}\right)^{N_{\mathrm{bf}}}
\end{aligned}
$$

whereas for the case with bang-bang control, it is

$$
\begin{aligned}
\Phi_{2 \mathrm{~N}_{\mathrm{bf}}}^{\mathrm{bb}}(x) & =\mathscr{F}^{-1}\left[\left(\mathscr{F}\left[\Phi_{2}^{\mathrm{bb}}\right]\right)^{N_{\mathrm{bf}}}\right] \\
& =\int_{-\pi}^{\pi} \frac{d k}{2 \pi \gamma^{2 N_{\mathrm{bfl}}}} e^{-i k x}\left(\frac{[1-\cos ((\gamma+1) k)]}{[1-\cos (k)]}\right)^{N_{\mathrm{bf}}}
\end{aligned}
$$

with $\mathscr{F}$ and $\mathscr{F}^{-1}$ denoting the discrete Fourier transformation and its inverse, respectively.

Already for random walk step-numbers on the order of 10, the resulting distributions are almost Gaussian. Their standard deviations give the rms deviations of the random walk models plotted in Fig. 3 . As in the numerical simulations at long times, they grow as a square-root of the number of steps. As one can recognize, the underlying two-step distributions in the $k$-space (i.e., the functions in the large brackets of Equs. (12) and (131)) are symmetric and differentiable around zero such that the above integrals can be evaluated analytically using the saddle point approximation (the small parameter is $k$, which is justified at least qualitatively in our bounded variable integral). We find for their variances in real space representation

$$
\Delta \sigma_{\mathrm{bfl}}\left(N_{\mathrm{bfl}}\right)=\sqrt{N_{\mathrm{bff}}} \beta=\sqrt{N_{\mathrm{bfl}}} \frac{\sqrt{5}}{2} \alpha \tau_{\mathrm{bfl}}
$$

for the case without bang-bang control and

$$
\Delta \sigma_{\mathrm{bb}}\left(N_{\mathrm{bff}}\right)=\frac{\sqrt{N_{\mathrm{bff}}}}{2} \gamma=\sqrt{\frac{N_{\mathrm{bff}}}{2}} \alpha \tau_{\mathrm{bb}}
$$

for the case with it. In the large- $N_{\mathrm{bff}}$ limit, this model shows excellent agreement with the numerical simulations.

At first sight, treating bang-bang pulses as $\delta$-function impulses appears to be an extraordinarily strong assumption, especially because in a physical implementation, the large bandwidth associated with very short pulses could excite other noise sources. However, this 
$\delta$-function impulse approximation is only for technical simplification. In fact, going to the other extreme of a wide, continuous pulse of the form $\sin \left(\frac{\pi}{\tau_{b b}} t\right)$ would also refocus our bflnoise over the course of its periods. Comparing the two-step deviation distributions arising from $\delta$-function impulses versus continuous sine waves, one obtains for the $\delta$-function case

$$
\Phi_{2}^{\inf }(x)=\frac{|\gamma-x|}{\gamma} \theta(\gamma-x) \theta(\gamma+x)
$$

and for the continuous sine wave case

$$
\Phi_{2}^{\text {cont }}(x)=\left\{\left[\frac{\pi}{2 \gamma}+\frac{\pi}{4 \gamma} \cos \left(2 \pi \frac{x}{\gamma}\right)\right]\left(1-\frac{x}{\gamma}\right)+\frac{3}{16 \gamma} \sin \left(2 \pi \frac{x}{\gamma}\right)\right\} \theta(\gamma-x) \theta(\gamma+x)(17
$$

These distributions are depicted in Fig. 6. One recognizes that in fact the distribution arising in the continuous sine wave case is narrower (and therefore indicates more effective noise suppression) than the $\delta$-function impulse case, with the drawback of leaving less free evolution time for coherent operation.

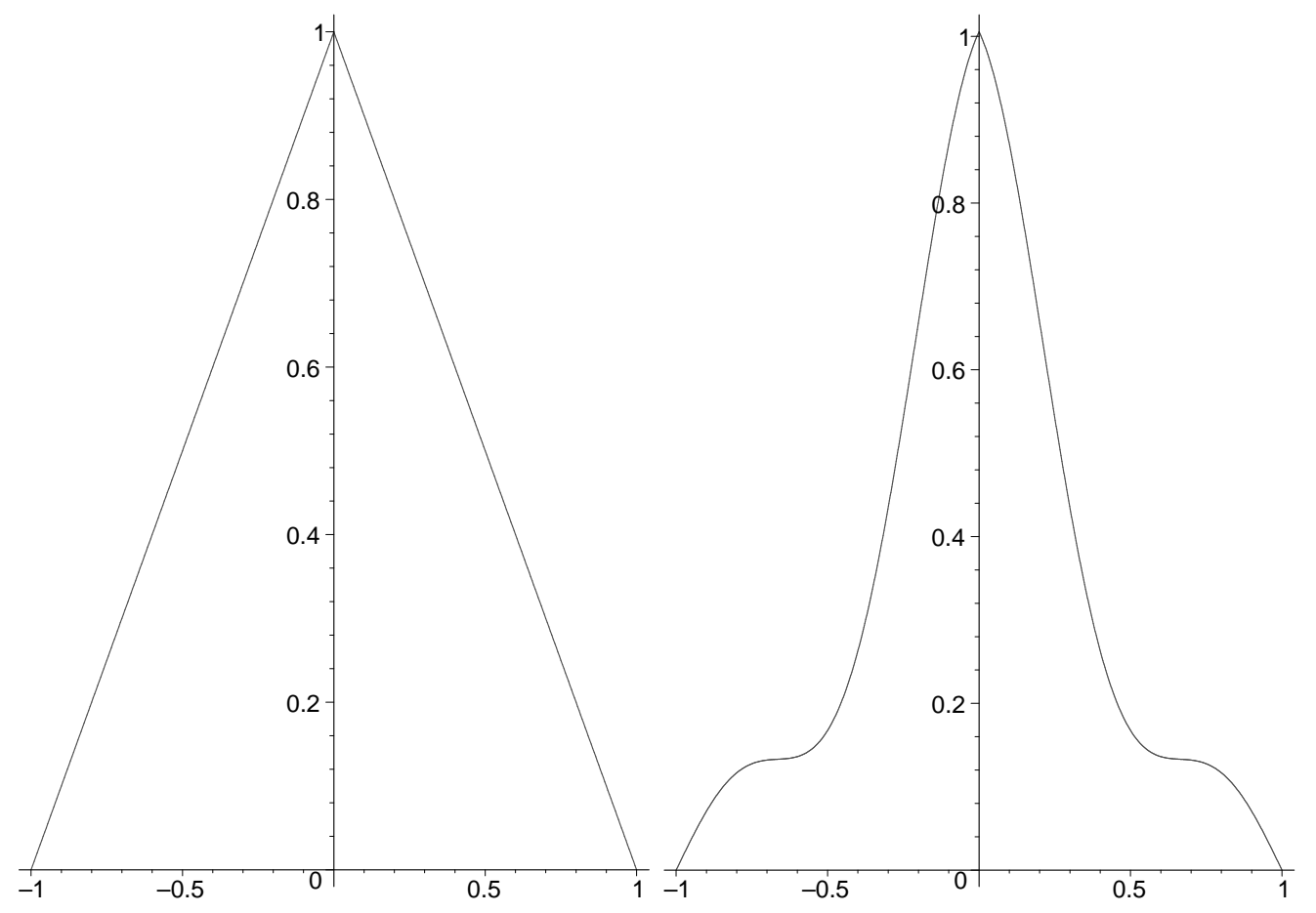

FIG. 6: Comparison of two-step distributions for the random walks with bang-bang control when the bang-bang pulses are taken to be $\delta$-functions (left) versus a continuous sine wave $\sin \left(\frac{\pi}{\tau_{b b}} t\right)$ (right). For clarity, the $y$-axis is rescaled to the maximum values of the distributions, while the $x$-axis is given in units of $\gamma$. 


\section{Distributions of the random walks deviation}

Beyond predicting the variances of the random walks, our analysis also allows evaluation of their full probability distributions. We compare them to numerics with and without bang-bang compensation by use of simulations with $10^{4}$ realizations at an evolution time $t_{0}=\tau_{\text {Sys }}$. The numerical histograms of the deviations with their respective one- and two-dimensional Gaussian fits are shown in Fig. 7.
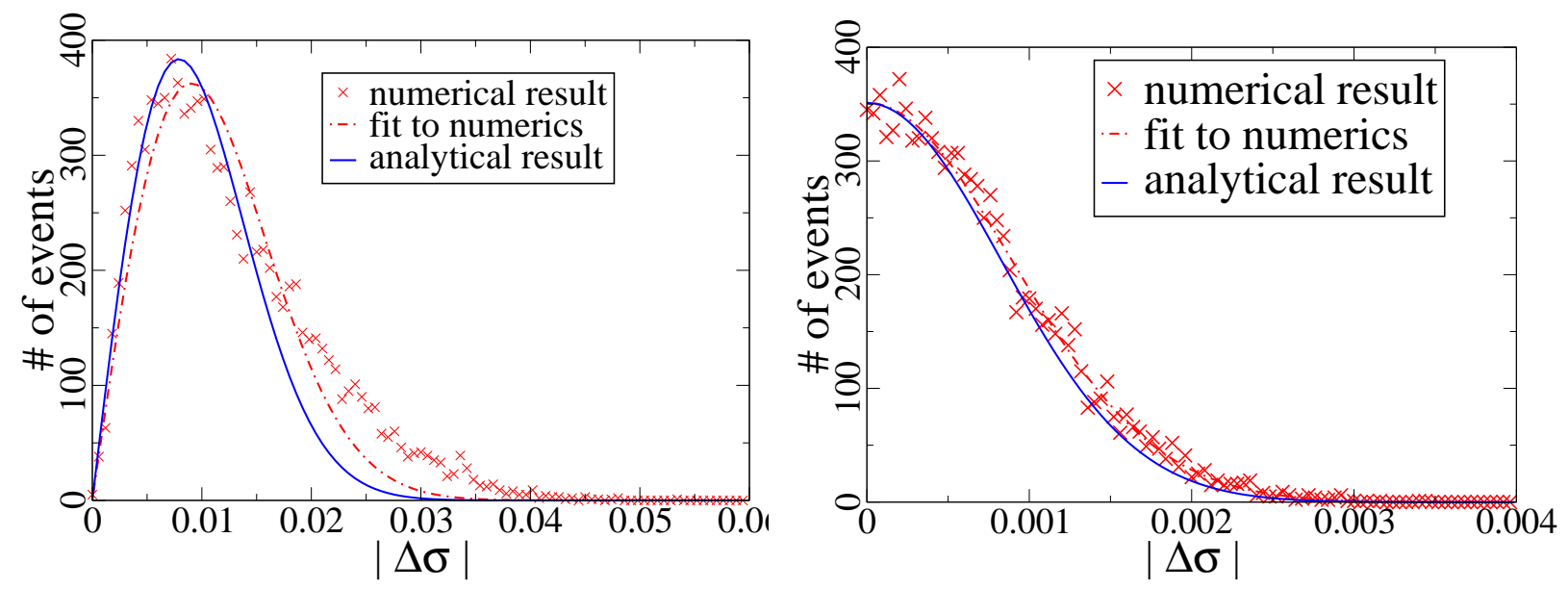

FIG. 7: Histograms of the deviation from free evolution both without bang-bang control (left) and with bang-bang control (right). Also plotted are fits to the expected two- and one-dimensional random walk statistics respectively associated with the uncontrolled and controlled cases. Numerical data were collected over $10^{4}$ realizations at a fixed time $t_{0}=\tau_{\text {Sys }}$ defined such that $\tau_{\text {bfl }}=0.01 \tau_{\text {Sys }}$ and thus $N_{\mathrm{bfl}}=\tau_{\mathrm{Sys}} / \tau_{\mathrm{bfl}}=100$ steps. (NB: The $x$-axis scale of the right graph depicting the bang-bang controlled case is 15 times smaller than that of the left graph depicting the uncontrolled case.)

We observe that not only the distribution obtained with bang-bang control is much narrower than the distribution obtained without it, but also that its shape is qualitatively different. The maximum of the bang-bang controlled distribution is at zero error. In contrast, the uncontrolled distribution has its maximum at a finite error $|\Delta \sigma|_{\max } \approx 0.01$, and it has zero probability of zero error. This reflects the one-dimensional nature of the bangbang controlled random walk in contrast to the two-dimensional nature of the uncontrolled random walk. 


\section{Bang-bang control working as a high-pass filter}

In order to measure the degree of noise suppression due to bang-bang control, we define the suppression factor $\mathcal{S}_{t_{0}}$ as follows for a given evolution time $t_{0}$

$$
\mathcal{S}_{t_{0}}\left(\tau_{\mathrm{bfl}} / \tau_{\mathrm{bb}}\right) \equiv \frac{\Delta \vec{\sigma}_{\mathrm{rms}}^{\mathrm{bfl}}\left(t_{0}\right)}{\Delta \vec{\sigma}_{\mathrm{rms}}^{\mathrm{bb}}\left(t_{0}\right)} .
$$

We now systematically study the dependence of $\mathcal{S}_{t_{0}}$ on $\tau_{\mathrm{bfl}} / \tau_{\mathrm{bb}}$ for a constant mean bfl switching rate $\tau_{\mathrm{bfl}}=10^{-2} \tau_{\text {sys }}$ at a fixed evolution time $t_{0}=\tau_{\text {sys }}$. The numerical data in Fig. 8 show that the suppression efficiency is linear in the bang-bang repetition rate, $S_{\tau_{\mathrm{sys}}}=\mu \tau_{\mathrm{bfl}} / \tau_{\mathrm{bb}}$. The numerically derived value of the coefficient, $\mu_{\text {numerical }} \approx 1.679$, is in excellent agreement with the analytical result $\mu_{\text {analytical }}=\sqrt{5 / 2} \simeq 1.581$ from our saddle point approximation, Equs. (14) and (15).

This small discrepancy between the numerical and analytical results is due to the fact that the analytical calculations neglect correlations between the parallel and perpendicular components of the random walk. This leads to an underestimate of the rms-deviation

$\Delta \vec{\sigma}_{\mathrm{rms}}^{\mathrm{bfl}}$ in the case without bang-bang control (compare also to Fig. (7). Therefore, we have quantitatively proved our qualitative intuition: bang-bang control affects the bfl noise signal like a high-pass filter, an effect that one of the authors has generally predicted for dynamical decoupling techniques [21].

\section{LIMITATIONS DUE TO PULSE INACCURACIES}

Thus far, we have tacitly assumed that one could apply perfect, zero-width $\pi$-pulses along exactly the $\hat{\sigma}_{\mathrm{x}}$-axis of the Bloch sphere. We now take into account that the control pulses themselves typically will have slight fluctuations in their duration or polarization that interfere with the desired refocusing. As already shown at the end of Section IV.B, the restriction of pulses to infinitesimal duration can be significantly relaxed. We now investigate to what extent the restriction to perfect pulses can be relaxed. 


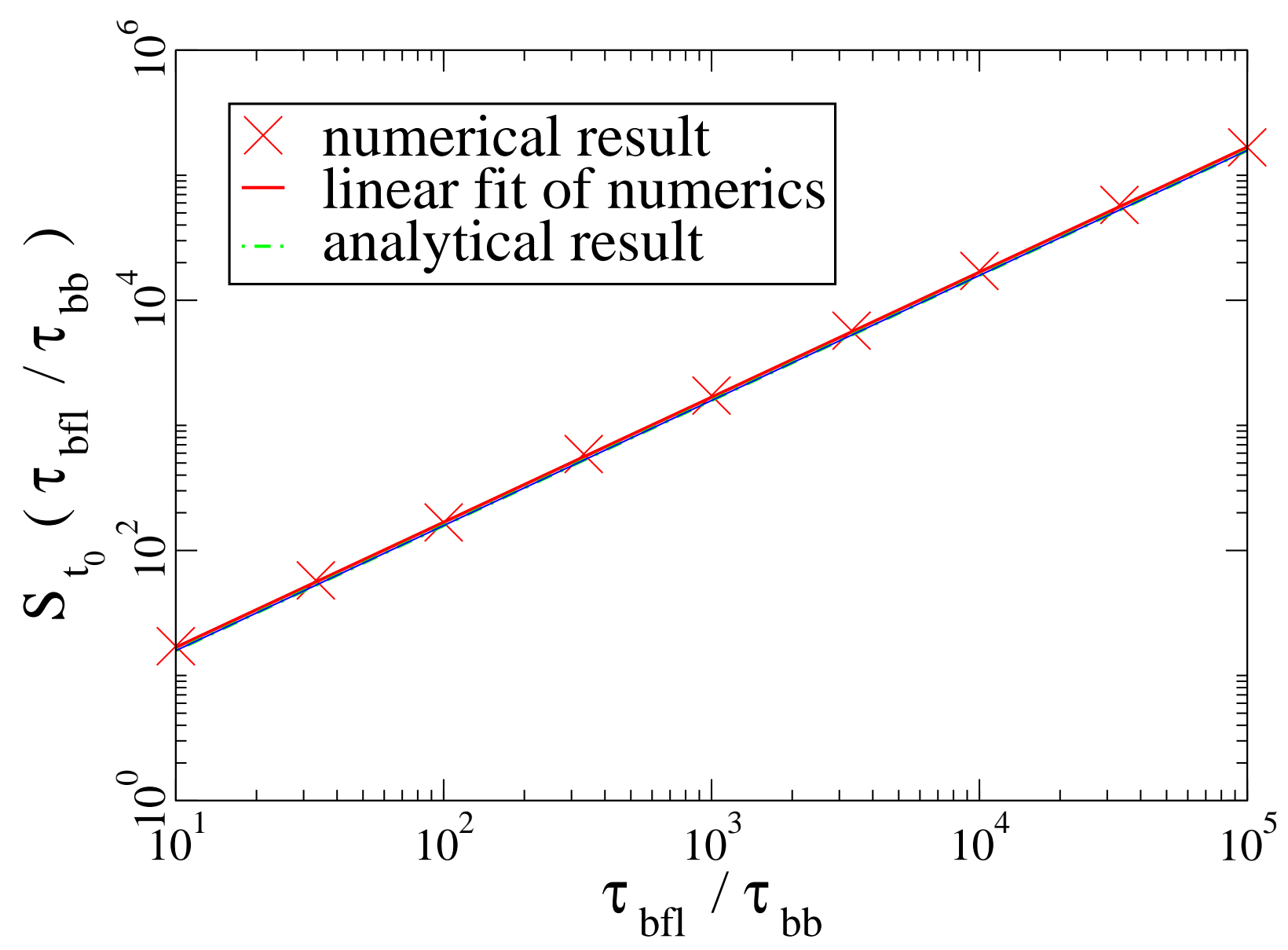

FIG. 8: The suppression factor $\mathcal{S}_{t_{0}}\left(\tau_{\mathrm{bfl}} / \tau_{\mathrm{bb}}\right)=\frac{\Delta \vec{\sigma}_{\mathrm{rms}}^{\mathrm{bf}}\left(t_{0}\right)}{\Delta \vec{\sigma}_{\mathrm{rms}}^{\mathrm{bb}}\left(t_{0}\right)}$ evaluated for $t_{0}=\tau_{\text {Sys }}$ as a function of the ratio of the mean switching time $\tau_{\mathrm{bfl}}$ and the bang-bang pulse separation $\tau_{\mathrm{bb}}$.

\section{A. Two generic types of bang-bang inaccuracies}

We essentially analyze two generic types of errors that could occur in the control apparatus when trying to apply $\pi$-pulses in $\hat{\sigma}_{\mathrm{x}}$-direction. One, the duration of each pulse could exhibit fluctuations, resulting in fluctuations in the rotation-angle around the desired value of $\pi$. Two, the polarization axis could suffer from directional deviations around the desired value of $\hat{\sigma}_{\mathrm{x}}$. Assuming the statistical independence of each pulse error, we expect for both types of imperfections a random-walk-like behavior of increasing deviations compared to evolutions with perfect pulses. 


\section{One-dimensional pulse error (dephasing)}

We make the quite general assumption that we may model the one-dimensional phase fluctuation of the imperfect bang-bang pulses $\phi_{j}(x)$ as a Gaussian distribution of the pulse durations and therefore of the rotation angles around their intended value $\pi$. This assumption should be valid for many physical situations, e.g., if the inaccuracy is due to electromagnetic noise in the pulse generator. The Gaussian is parameterized by its standard deviation $\delta \phi_{0}$ (see Fig. (9). Thus, the corresponding pulse angle aberration of the $j$ th step is given by

$$
\phi_{j}^{1 \mathrm{~d}}(x)=\frac{1}{\sqrt{2 \pi} \delta \phi_{0}} e^{-\frac{x^{2}}{2 \delta \phi_{0}^{2}}} .
$$

Having assumed a Gaussian distribution, we can exactly evaluate the distributions of the $\mathrm{N}$ step deviation $\Delta \Phi_{N}$ (which are usually given as $N$-fold time-convoluted integrals) as follows by use of the convolution theorem

$$
\begin{aligned}
\Phi_{N}^{1 \mathrm{~d}} & =\mathscr{F}^{-1}\left[\Pi_{j=1}^{N} \tilde{\phi}_{j}\right] \\
& =\frac{1}{\sqrt{2 \pi N} \delta \phi_{0}} e^{-\frac{x^{2}}{2 N \delta \phi_{0}^{2}}}
\end{aligned}
$$

with $\tilde{\phi}_{j}^{1 \mathrm{~d}}=\mathscr{F}\left[\phi_{j}\right]$ denoting the Fourier transform of $\phi_{j}^{1 \mathrm{~d}}$ and $\mathscr{F}^{-1}$ denoting the inverse Fourier transform.

Therefore the rms displacement in the random walk increases as a square-root in the number $N$ of bang-bang pulses: $\delta \phi_{N}=\sqrt{N} \delta \phi_{0}$. Equivalently, the dephasing grows as square-root in time

$$
\delta \phi(t)=\sqrt{t / \tau_{\mathrm{bb}}} \delta \phi_{0}
$$

on the time scale of our coarse-graining (which is here given as $\tau_{\mathrm{bb}}$ ).

\section{Two-dimensional pulse error: dephasing and relaxation/excitation}

A similar argument works when there are also fluctuations around the desired $\hat{\sigma}_{\mathrm{x}}$ rotation axis. Each individual variation of the axis can be split into two components: (1) $\delta \phi_{\text {perp }}$, which is perpendicular to the connecting vector between the $\hat{\sigma}_{\mathrm{x}}$-axis and the qubit state $\vec{\sigma}(t)$ on the Bloch sphere, and (2) $\delta \phi_{\text {tan }}$, which is transverse to it (see Fig. 10). To first order, the 


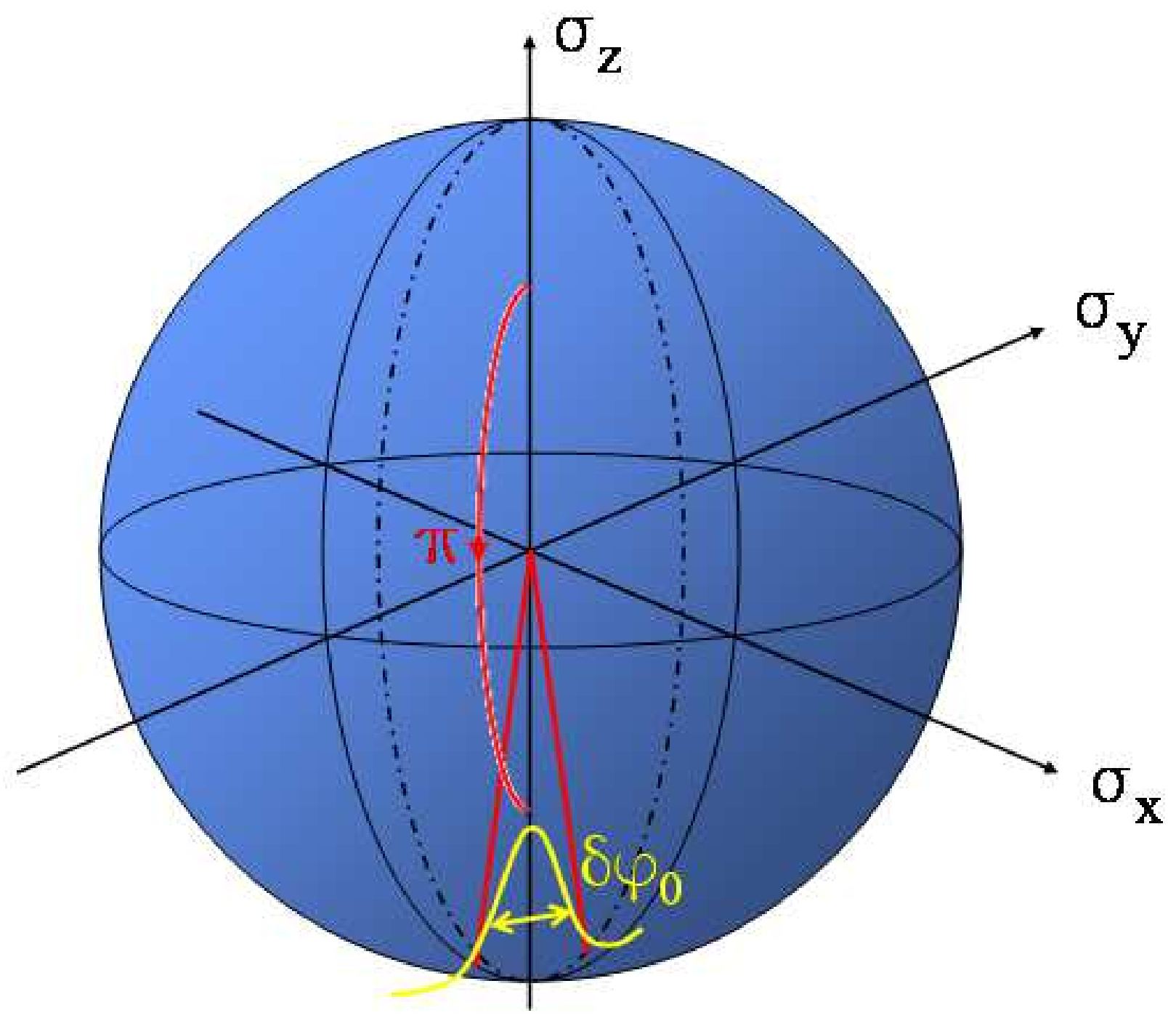

FIG. 9: Sketch of one-dimensional bang-bang aberration. The variations $\delta \phi_{0}$ of the rotation angle around the desired value of $\pi$ leads to slight deviations in parallel to the permitted dynamical direction, thus generating dephasing.

perpendicular part does not disturb the intended spin-flip [33]. However, the transverse part does cause a deviation from the ideal spin-flip in a direction toward or away from the previous qubit state. (Therefore, it produces relaxation or excitation, as its effect is orthogonal to the free $\hat{\sigma}_{\mathrm{x}}$-evolution.) Consequently, in a statistical average we only have to consider $1 / \sqrt{2}$ of the typical total mean $\delta \phi_{0}$ of the aberration. The effect of a $\pi$-rotation around an axis tilted by an angle $\delta \phi_{\tan }$ is a deviation $2 \delta \phi_{\tan }$ from the trajectory of the perfect evolutions; thus we receive altogether a deviation on the order of $\sqrt{2} \delta \phi_{0}$. 


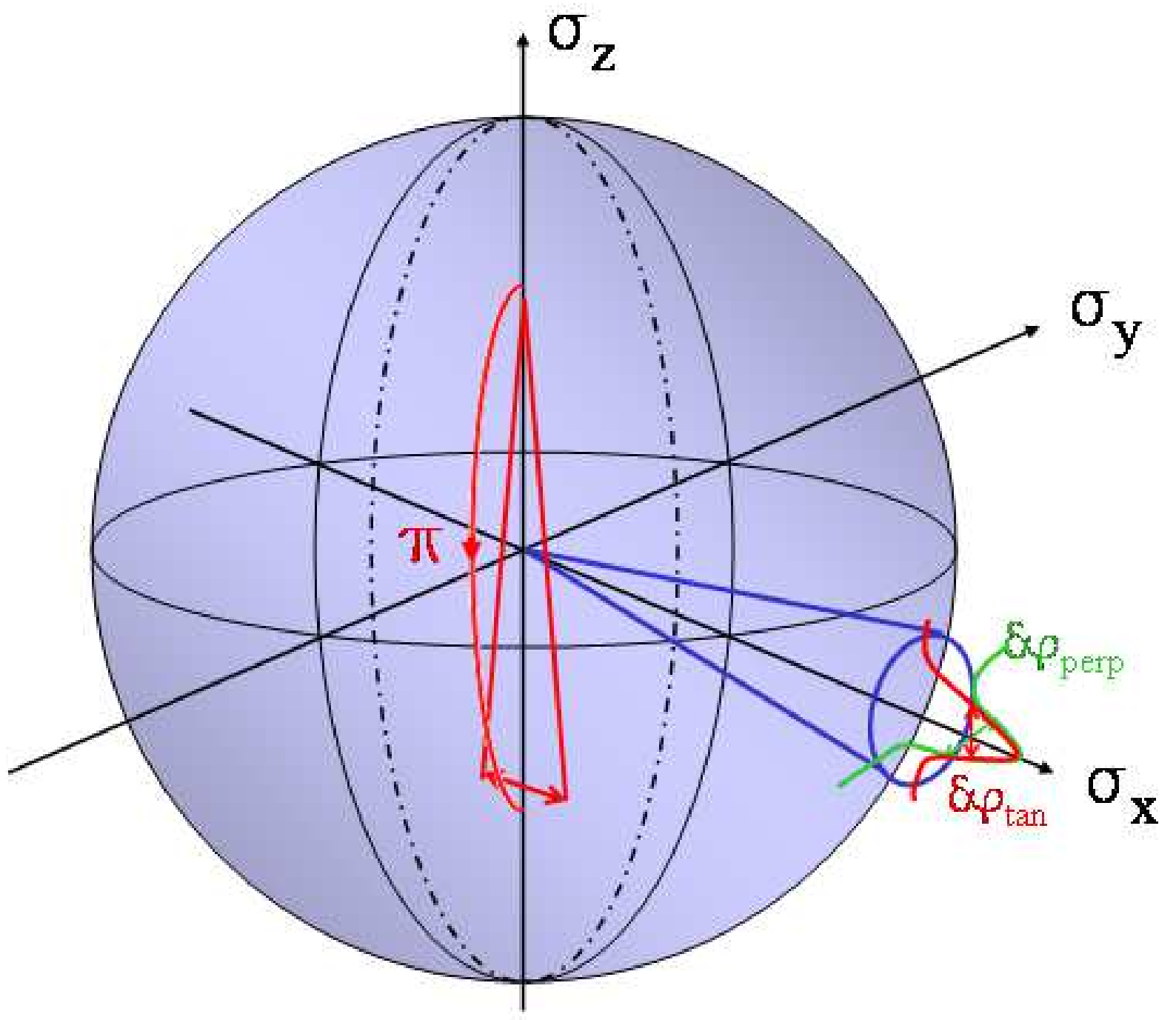

FIG. 10: Sketch of two-dimensional bang-bang aberration. To first order, variations $\delta \phi_{\text {perp }}$ of the rotation axis perpendicular to the connection vector between $\sigma_{\mathrm{x}}$ and the qubit state (here for simplicity: $\sigma_{\mathrm{z}}=+1$ ) do not influence the intended spin-flip, whereas the variations $\delta \phi_{\tan }$ along this line causes deviations on the Bloch sphere perpendicular to the permitted evolution trajectories (therefore producing relaxation or excitation).

Therefore, we obtain analogously to Equ. (19) for each single step distribution

$$
\phi_{j}^{2 \mathrm{~d}}(x)=\frac{1}{\sqrt{2 \pi} \sqrt{2} \delta \phi_{0}} e^{-\frac{x^{2}}{4 \delta \phi_{0}^{2}}} ;
$$

and analogously to Equ. (20) for the deviation after $N$ steps

$$
\Phi_{N}^{2 \mathrm{~d}}=\frac{1}{\sqrt{2 \pi} \sqrt{2 N} \delta \phi_{0}} e^{-\frac{x^{2}}{4 N \delta \phi_{0}^{2}}}
$$


Equivalently, in terms of the time $t$

$$
\delta \phi(t)=\sqrt{2 t / \tau_{\mathrm{bb}}} \cdot \delta \phi_{0}
$$

\section{B. Numerical and analytical results}

In the same manner as our previous integrations of a stochastic Schrödinger equation, we numerically simulate qubit dynamics under inaccurate pulses. In the first instance, we work without bfl-noise to verify our analytical random walk model. Later, we add the bfl-noise in order to study the competition between the two sources of error.

\section{Random walk due to inaccurate bang-bang pulses only}

We analyze deviations on the Bloch sphere between the noiseless case trajectories that occur when the bang-bang pulses are perfect and those when they are not. As per Equ. (5), we calculate the rms-deviation over ensembles of $N=10^{3}$ realizations. As a representative time point, we once again choose $t_{0}=\tau_{\text {Sys }}$. This is because, as explained in the discussion surrounding Fig. 3, this time scale should exhibit neither short-time effects nor near-total decoherence. From Equs. (21) and (24), it immediately follows that for the mean deviations at $t_{0}$ if there are phase errors

$$
\Delta \sigma_{\mathrm{bb}}^{1 \mathrm{~d}}\left(t_{0}\right)=\sqrt{N_{\mathrm{bb}}} \delta \phi_{0}=\sqrt{\frac{t_{0}}{\tau_{\mathrm{bb}}}} \delta \phi_{0},
$$

and if there are axis errors

$$
\Delta \sigma_{\mathrm{bb}}^{2 \mathrm{~d}}\left(t_{0}\right)=\sqrt{2 N_{\mathrm{bb}}} \delta \phi_{0}=\sqrt{2 \frac{t_{0}}{\tau_{\mathrm{bb}}}} \delta \phi_{0} .
$$

As characteristic values for the mean accuracy of single pulses, we choose $\delta \phi_{0}$ in the range of $10^{-6}$ to $10^{-4}$, which should be technologically feasible. As one can see in the double logarithmic plots of Fig. 11, the numerically determined evolutions follow the analytically expected square-root type random walk behavior. 

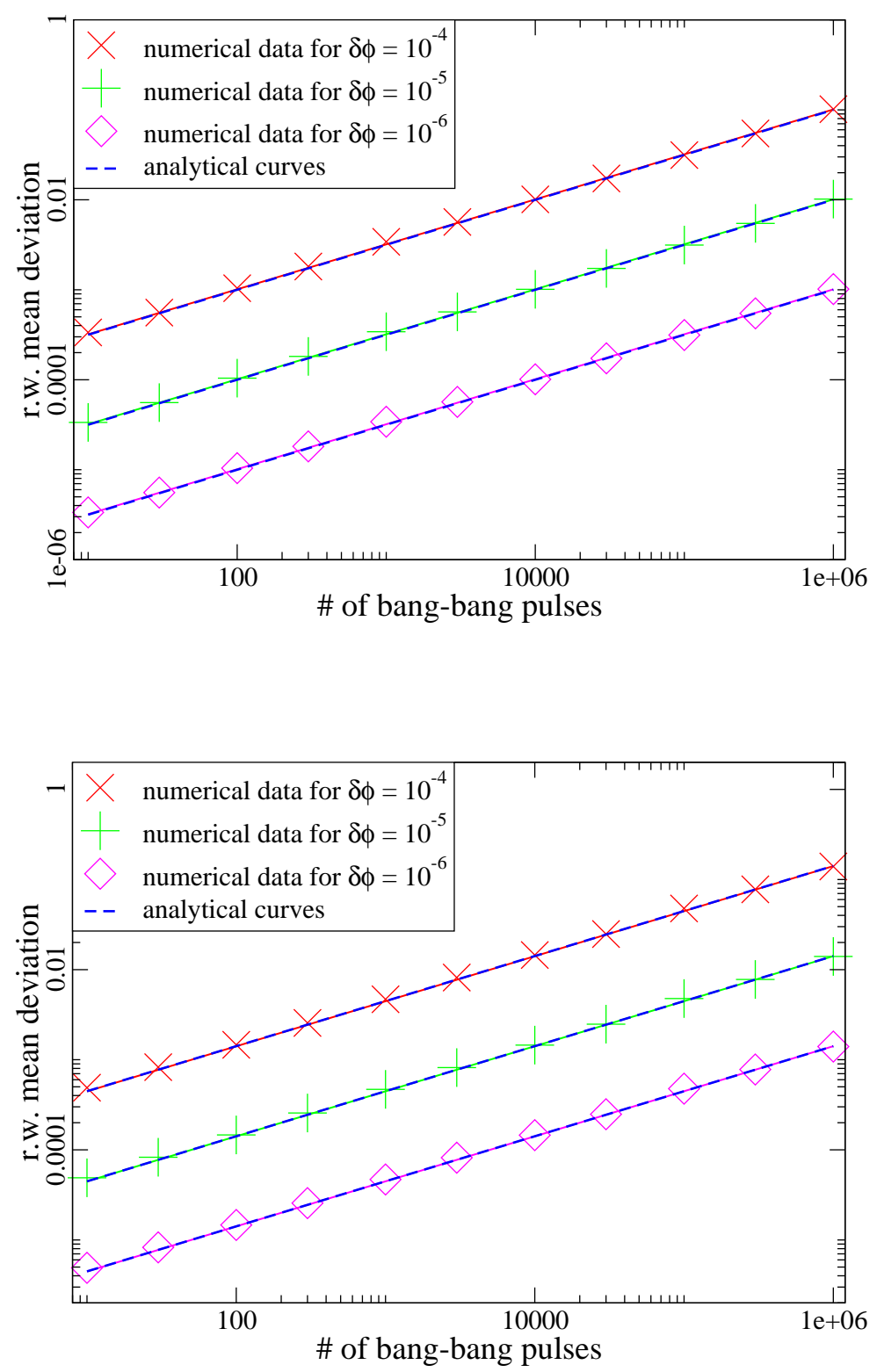

FIG. 11: Plot of the one- respectively two-dimensional imperfectly bang-bang pulsed evolution. Dashed lines are square-root fits of the numerical data, while the solid lines denotes the analytical calculations.

\section{Random walk due to both inaccurate bb-pulses and bfl-noise}

We now combine our imperfect bang-bang pulse operations with our former bfl-noise signal to discuss the applicability of our control scheme when "realistic" pulse generators are used. As before, we calculate the rms deviations at $t_{0}=\tau_{\text {Sys }}$ by averaging over $10^{3}$ realizations. The bfl-parameters are those used previously: a coupling strength $\alpha=0.1$ and 
an average switching time $\tau_{\mathrm{bfl}}=0.01 \tau_{\text {Sys }}$. However, with the aim of determining the optimal bang-bang protocol in the presence of pulse imperfections, we now consider different pulse separation times $\tau_{\mathrm{bb}} / \tau_{\text {Sys }}$ ranging from $10^{-5}$ to $10^{-2}$.

We assume that the errors induced by the bfl and those induced by the pulse generator are statistically independent, and thus we sum together both sets of induced deviations in the usual rms-fashion. In comparison to the case of ideal bang-bang pulses, Equ. (15), we find here the average total deviations induced by both bfl telegraph noise and imperfect bang-bang pulses to be:

$$
\begin{aligned}
\Delta \sigma_{\mathrm{tot}}^{1 d} & =\sqrt{\Delta \sigma_{\mathrm{bfl}}^{2}+\Delta \sigma_{\mathrm{bb}}^{1 d^{2}}} \\
& =\sqrt{\frac{1}{2} N_{\mathrm{bfl}} \alpha^{2} \tau_{\mathrm{bb}}^{2}+N_{\mathrm{bb}} \delta \phi_{0}^{2}} \\
& =\sqrt{\frac{1}{2} \alpha^{2} \tau_{\mathrm{bb}}^{2} \frac{t_{0}}{\tau_{\mathrm{bfl}}}+\delta \phi_{0}^{2} \frac{t_{0}}{\tau_{\mathrm{bb}}}}
\end{aligned}
$$

in the one-dimensional case where imperfect pulses only impart phase errors (due to imprecise pulse duration), and

$$
\begin{aligned}
\Delta \sigma_{\mathrm{tot}}^{2 d} & =\sqrt{\Delta \sigma_{\mathrm{bfl}}^{2}+\Delta \sigma_{\mathrm{bb}}^{2 d^{2}}} \\
& =\sqrt{\frac{1}{2} N_{\mathrm{bfl}} \alpha^{2} \tau_{\mathrm{bb}}^{2}+2 N_{\mathrm{bb}} \delta \phi_{0}^{2}} \\
& =\sqrt{\frac{1}{2} \alpha^{2} \tau_{\mathrm{bb}}^{2} \frac{t_{0}}{\tau_{\mathrm{bff}}}+2 \delta \phi_{0}^{2} \frac{t_{0}}{\tau_{\mathrm{bb}}}}
\end{aligned}
$$

in the two-dimensional case when imperfect pulses impart both phase and relaxation/excitation errors (due to imprecision in the pulses' polarization axes).

As Fig. 12 demonstrates, we observe a very good agreement between our numerical and analytical results. Such data make it possible to determine an optimal bang-bang separation time $\tau_{\mathrm{bb}}^{\mathrm{opt}}$. Specifically, this optimum can be derived by calculating the zero value of the derivative of Equs. (27) and (28) with respect to $\tau_{\mathrm{bb}}$. We therefore conclude that the optimal period between bang-bang pulses is:

$$
\tau_{\mathrm{bb}}^{1 d}=\sqrt[3]{\tau_{\mathrm{bfl}} \frac{\delta \phi_{0}^{2}}{\alpha^{2}}}
$$

for the one-dimensional case and

$$
\tau_{\mathrm{bb}}^{2 d}=\sqrt[3]{2 \tau_{\mathrm{bf}} \frac{\delta \phi_{0}^{2}}{\alpha^{2}}}
$$



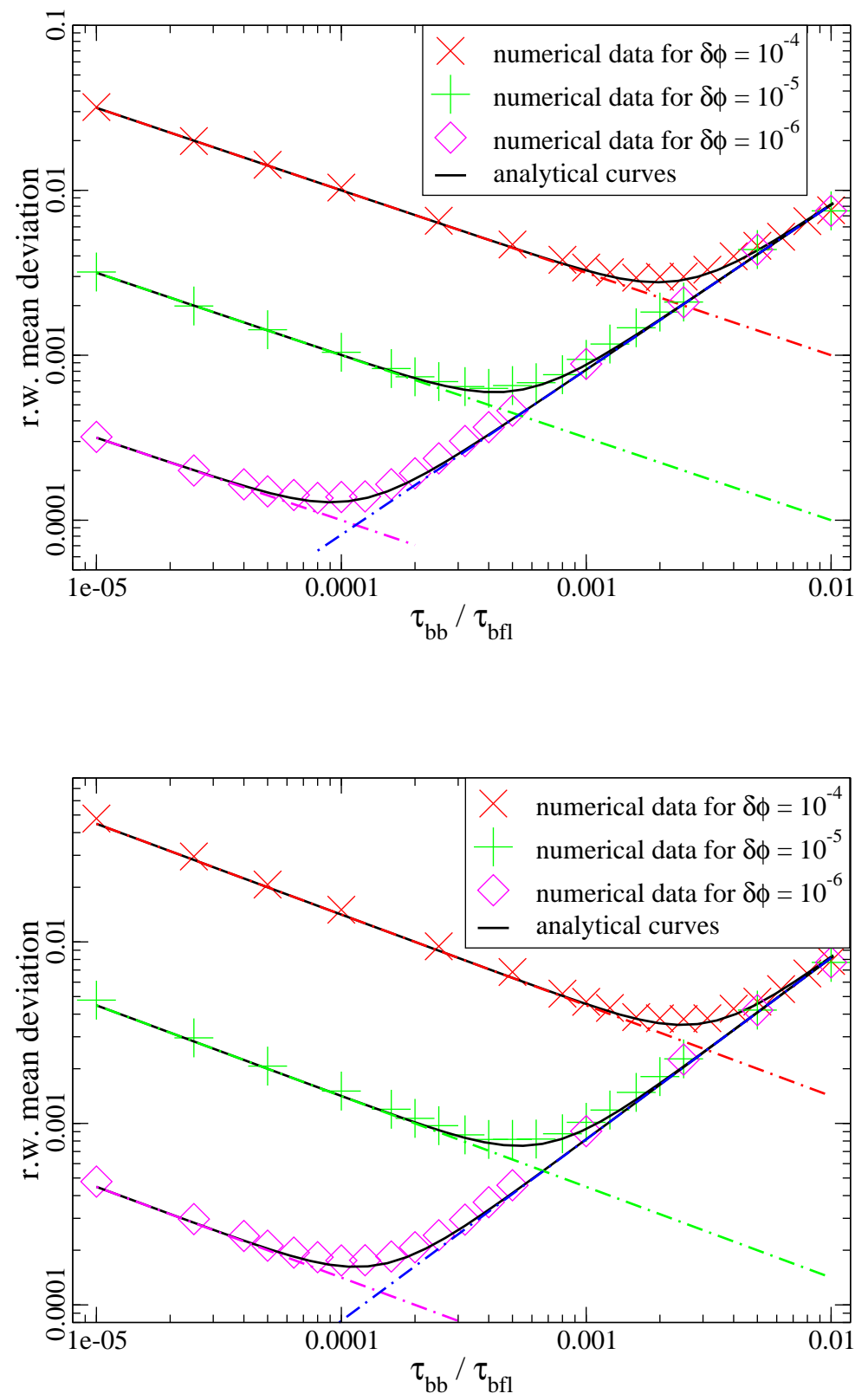

FIG. 12: Plot of the Bloch sphere rms deviations received by one-/two-dimensional inaccurately pulsed bang-bang compensation of the typical bfl-perturbation. Dashed lines describe the aberrances for pure faulty bang-bang (i.e. without bfl-noise), respectively the exactly compensated bfl-case (see Fig. 31), while solid lines denotes the deviations calculated by random walk analysis.

for the two-dimensional case. These optimal times respectively correspond to minimized variances at $t_{0}=\tau_{\text {Sys }}$ of

$$
\Delta \sigma_{\mathrm{opt}}^{1 d}=\sqrt{\frac{1}{2}+1} \frac{\alpha^{1 / 3} \delta \phi_{0}^{2 / 3}}{\tau_{\mathrm{bfl}}^{1 / 6}} \sqrt{t_{0}}
$$


for the one-dimensional case of only imprecise pulse durations and

$$
\Delta \sigma_{\mathrm{opt}}^{2 d}=\sqrt{2^{-1 / 3}+2^{2 / 3}} \frac{\alpha^{1 / 3} \delta \phi_{0}^{2 / 3}}{\tau_{\mathrm{bfl}}^{1 / 6}} \sqrt{t_{0}}
$$

for the two-dimensional case of both imprecise pulse durations and polarization axes.

\section{CONCLUSION AND REMARKS}

We have investigated the qubit errors that arise from the noise generated by a single bistable fluctuator (bfl) in its semiclassical limit, where it behaves as a telegraph noise source. We numerically integrated a corresponding stochastic Schrödinger equation, Equ. (4), as well as analytically solved (in the long-time limit) appropriate random walk models. As a characteristic measure of the resulting dephasing and relaxation effects, we used the rms deviation of noisy evolutions compared to noiseless ones. To suppress the effects of this noise, we presented a bang-bang pulse sequence analogous to the familiar spin-echo method. We claimed this pulse sequence to be capable of refocusing most of the bfl-noise induced aberrations. Both in the case without bang-bang control and the case with it, there was excellent agreement between our numerical and analytical results on the relevant intermediate time scales (i.e., times after a short initial phase where deviations grow linearly instead of as a square-root in time, but before the qubit becomes totally decohered).

In particular, we confirmed our preliminary qualitative picture that bang-bang control works as a high pass filter, suppressing qubit errors by a factor $\mu \tau_{\mathrm{bfl}} / \tau_{\mathrm{bb}}$ that is directly proportional to the ratio of the mean bfl switching time and the period between bang-bang pulses. The numerically and analytically calculated constants of proportionality $\mu_{\text {numerical }} \approx$ 1.679 and $\mu_{\text {analytical }}=\sqrt{5 / 2} \simeq 1.581$ also matched to good accuracy. These results imply that the bang-bang procedure is an appropriate remedy against the $1 / f$-noise that often is seen in solid-state environments. This is because bang-bang control exhibits maximal suppression of bfl telegraph noise, and $1 / f$ noise generally arises from an ensemble of bfls. Finally, one has to be aware that also the static $\sigma_{\mathrm{z}}$-term of the Hamiltonian is averaged out, and this generally reduces the degree of control on the qubit. But this is only a technical constraint, as one could imagine interchanging two different types of bang-bang pulses (e.g. along the $x$ and the $y$-axis respectively) to admit corresponding quantum-gate operations.

We previously presented this basic idea in a short paper [27]. The present work extends 
that short paper by treating the effects of different types on non-ideal bang-bang pulses. Moreover, the analytical random walk method is outlined in much more detail, as this method should also be applicable to other problems that are difficult to treat in a master equation approach.

Meanwhile, several other extensions of Ref. 27] have been proposed by other research groups. Ref. [17] includes a larger number of fluctuators, described as semiclassical noise sources, but restricts itself to a single spin-echo cycle. Ref. 28] analyzes extensively the importance of higher, non-Gaussian cumulants and memory effects and arrives at a number of analytical results, but it does not treat the option of refocusing. Ref. 22] treats a full microscopic model and compares different variations of the bang-bang pulse sequence. Ref. 30] also treats a full microscopic model with potentially many fluctuators using a Lindblad-type approach and covers a wide range of ratios between the fluctuator and bang-bang pulse time scales. One of its main conclusions is that a Zeno effect is found in a parameter regime not covered by our work. Note that all of these other extensions of our work treat only the case of ideal bang-bang pulses.

\section{Acknowledgments}

We are indebted to G. Falci, I. Goychuk, T.P. Orlando and J. von Delft for helpful discussions. Our special thanks goes to A. Kaeck for pointing out the capability of our random walk model to describe deviations due to imperfect pulsing. HG and FKW also thank T.P. Orlando for his great hospitality during their stay at MIT. WMK gratefully acknowledges fellowship support from the Fannie and John Hertz Foundation. This work was supported by a DAAD-NSF travel grant and by the National Security Agency (NSA) and Advanced Research and Development Activity (ARDO) under Army Research Office (ARO) contract P-43385-PH-QC as well as by the DFG through SFB 631.

\section{APPENDIX A}

We shall now connect the model of a single bfl as a telegraph noise source to a microscopic Hamiltonian. We start with the conventional Hamiltonian model of a single bfl, e.g., 14, 31, 32]. The original qubit is influenced by noise from another qubit, the bfl, which itself is 
coupled to a thermal environment by a bilinear spin-boson type interaction:

$$
H=H_{\text {qubit }}+H_{\text {qubit,bfl }}+H_{\mathrm{bfl}}+H_{\mathrm{bfl}, \mathrm{env}}+H_{\mathrm{env}} \cdot
$$

where

$$
\begin{aligned}
H_{\mathrm{qubit}} & =\hbar \epsilon_{\mathrm{q}} \hat{\sigma}_{\mathrm{z}}^{\mathrm{q}}+\hbar \Delta_{\mathrm{q}} \hat{\sigma}_{\mathrm{x}}^{\mathrm{q}} \\
H_{\mathrm{qubit}, \mathrm{bfl}} & =\hbar \alpha \hat{\sigma}_{\mathrm{z}}^{\mathrm{q}} \hat{\sigma}_{\mathrm{z}}^{\mathrm{bfl}} \\
H_{\mathrm{bfl}} & =\hbar \epsilon_{\mathrm{bfl}} \hat{\sigma}_{\mathrm{z}}^{\mathrm{bfl}}+\hbar \Delta_{\mathrm{bfl}} \hat{\sigma}_{\mathrm{x}}^{\mathrm{bfl}} \\
H_{\mathrm{bfl}, \mathrm{env}} & =\hbar \lambda \hat{\sigma}_{\mathrm{z}}^{\mathrm{bfl}} \sum_{j}\left(\hat{a}_{j}^{\dagger}+\hat{a}_{j}\right) \\
H_{\mathrm{env}} & =\hbar \sum_{j} \omega_{j}\left(\hat{a}_{j}^{\dagger} \hat{a}_{j}+11 / 2\right)
\end{aligned}
$$

The scalar $\alpha$ denotes the coupling strength between the original qubit and the bfl, while the scalar $\lambda$ indicates the influence of the environmental heat bath on the bfl.

It is not obvious how to treat such a combined open quantum system [14, 32]. The common approach of deriving a master equation for the reduced qubit system does not work, as it is not clear how to introduce an open quantum system "bfl" as the environment. Gassmann, et al. present four alternative approaches [14]. Their first approach is to derive a standard Markovian master equation for the combined open system "qubit + bfl" and trace out the parameters of the bfl afterwards. Their second approach is to consider the qubit as influenced by an effective bfl-bath environment by use of an Markovian and secular approximation in the limit of small $\alpha$. Their third approach, which is both the most general and the most complicated, is to deduce a master equation by applying a non-Markovian weak-coupling perturbation ansatz in second order in $\alpha$.

For our investigations, we prefer this last and most general approach: a stochastic treatment employing an appropriate randomly changing bfl-noise Hamiltonian term (compare also to [17]). This choice is not only because of practical reasons (to make our numerics feasible), but also due to empirical considerations (see [8, 9], where characteristics of telegraph noise were observed and attributed to bfls). Hence, we restrict our analysis to the limit $\lambda \gg \alpha$, i.e., the limit where the coupling of the bfl to the external environment is much larger than interaction between the bfl and the qubit. For convenience, we assume the bfl is in its high-temperature limit. (Note that this does not necessarily mean the qubit is also in a high-temperature regime for the qubit's energy scale might be much larger than that 
of the bfl.) We thus assume the bfl behaves like a classical (i.e., decohered) noise source, and we specifically describe the bfl's influence on the qubit with the following stochastic Hamiltonian:

$$
H_{\text {qubit,bfl }} \stackrel{\text { semicl. }}{\longrightarrow} H_{\text {qubit,bfl }}^{\text {noise }}(t)=\hbar \alpha \hat{\sigma}_{\mathrm{z}}^{\mathrm{q}} \xi_{\mathrm{bfl}}(t)
$$

In the equation above, $\xi_{\mathrm{bfl}}(t)$ is a random function of time representing the switching of the $\sigma_{\mathrm{z}}^{\mathrm{bfl}}$-value between \pm 1 . In our high-temperature limit, we assume $\xi_{\mathrm{bfl}}(t)$ has symmetrical Poissonian statistics (i.e., the probabilities of the bfl switching from +1 to -1 and from -1 to +1 are equal and constant over time). Such a symmetrical random process is readily described by just one parameter: the typical time separation $\tau_{\text {bfl }}$ between two bfl flips (see Fig. (1).

The high temperature limit is not a crucial constraint. Treating the strongly thermally coupled bfl in an intermediate temperature regime would only result in some asymmetrically switching $\xi_{\mathrm{bfl}}(t)$. The typical switching times time $\tau_{\mathrm{bfl}}^{\uparrow, \downarrow}$ for switching the bfl up and down respectively satisfy the detailed balance relation $\frac{\tau_{\mathrm{bA}}^{\downarrow}}{\tau_{\mathrm{bfl}}^{\uparrow}}=e^{-\delta E_{\mathrm{bff}} / k_{b} T}$, where $\delta E_{\mathrm{bfl}}$ denotes the energy separation of the two bfl-states, and $T$ the temperature of the heat bath which drives the switching of the bfl. The microscopic structure of the rates depends on details of the experiment. Typically, they will be golden rule rates containing the density of states of the heat bath and the matrix element of its coupling to the bfl. If that bath is made of harmonic oscillators with an ohmic spectral density, we e.g. expect switching rater $\left(\tau_{\mathrm{bfl}}^{\uparrow, \downarrow}\right)^{-1}= \pm \frac{\alpha_{0} \delta E_{\mathrm{bA}}}{e^{ \pm \delta E_{\mathrm{bff}} / k_{b} T}-1}$, where $\alpha_{0}$ is the dimensionless coupling strength to the Ohmic bath. This would essentially only lead to an additional drift of the qubit state, i.e., a random walk with a nonzero average value. Neither our analytical results nor our conclusions would otherwise change qualitatively. In fact, assuming the the bang-bang pulse cycles are sufficiently short relative both the typical +1 to -1 and -1 to +1 switching times of the bfl, bang-bang suppression of the bfl noise should not be diminished at all by the bfl's asymmetrical switching. We therefore obtain Equ. (11) as our starting point of the bfl-perturbed qubit dynamics.

[1] D. Loss and D. P. DiVincenzo, Phys. Rev. A 57, 120 (1998).

[2] D. Vion et al., Science 296, 886 (2002). 
[3] I. Chioresco, Y. Nakamura, C.J.P.M. Harmans, and J.E. Mooij, Science 299, 1869 (2003).

[4] J.M. Martinis, S.W. Nam, J. Aumentado, and C. Urbina, Phys. Rev. Lett. 89, 117907 (2002).

[5] Y. Nakamura, Y.A. Pashkin, and J.S. Tsai, Nature 398, 786 (1999).

[6] Yu. Makhlin, G. Schön, and A. Shnirman, Rev. Mod. Phys. 73, 357 (2001).

[7] C. van der Wal, F. Wilhelm, C. Harmans, and J. Mooij, Eur. Phys. J. B 31, 111 (2003).

[8] R. Wakai and D. van Harlingen, Phys. Rev. Lett. 58, 1687 (1987).

[9] N. M. Zimmermann, J. L. Cobb, and A. F. Clark, Phys. Rev. B 56, 7675 (1997).

[10] M. B. Weissmann, Rev. Mod. Phys. 60, 537 (1988).

[11] A. J. Leggett et al., Rev. Mod. Phys. 59, 1 (1987).

[12] Y. Makhlin, G. Schön, and A. Shnirman, Phys. Scr. T 102, 147 (2002).

[13] U. Weiss, Quantum Dissipative Systems (World Scientific, Singapore, 2001).

[14] H. Gassmann, F. Marquardt, and C. Bruder, Phys. Rev. E 66, 041111 (2002).

[15] N. Prokov'ef and P. Stamp, Rep. Prog. Phys. 63, 669 (2000).

[16] T. Ytakura and Y. Tokura, Dephasing due to background charge fluctuations, ISSP Int. Workshop "Quantum transport in mesoscopic scale and low dimensions", (2003).

[17] Y. M. Galperin, B. L. Altshuler, and D. V. Shantsev, Low-frequency noise as a source of dephasing a qubit, Proc. of NATO/Euresco Conf. "Fundamental problems of mesoscopic physics: Interaction and decoherence", Granada, Spain, (2003), NATO Science Series.

[18] P. Dutta and P. M. Horn, Rev. Mod. Phys. 53, 497 (1981).

[19] J. M. Martinis et al., Phys. Rev. B 67, 094510 (2003).

[20] S. Lloyd and L. Viola, Phys. Rev. A 58, 2733 (1998).

[21] S. Lloyd, E. Knill, and Viola, Phys. Rev. Lett. 82, 2417 (1999).

[22] S. Lloyd, E. Knill, and L. Viola, Phys. Rev. Lett. 83, 4888 (1999).

[23] L. Arnold, stochastische Differentialgleichungen (Oldenbourg, München, 1973).

[24] N. G. van Kampen, Stochastic processes in physics and chemistry (Elsevier, Amsterdam, 1997).

[25] H. Carr and E. Purcell, Phys. Rev. 94, 630 (1954).

[26] G. H. Weiss, Aspects and Applications of the Random Walk (North-Holland, Amsterdam, 1994).

[27] H. Gutmann, F.K. Wilhelm, W.M. Kaminsky, and S. Lloyd, cond-mat/0308107,

[28] K. Rabenstein, V. A. Sverdlov, and D. V. Averin, JETP Vol. 79, is. 12, 783 (2004). 
[29] L. Faoro and L. Viola, quant-ph/0312159.

[30] G. Falci, A. D'Arrigo, A. Mastellone, and E. Paladino, cond-mat/0312442.

[31] E. Paladino, L. Faoro, and G. Falci, cond-mat/0312411.

[32] E. Paladino, L. Faoro, G. Falci, and R. Fazio, Phys. Rev. Lett. 88, 228304 (2002).

[33] The attentive reader might object that a spin-flip around a different axis on the xy-equator doesn't commute with pure $\hat{\sigma}_{\mathrm{x}}$-dynamics, but rather with something nearby. As we do not

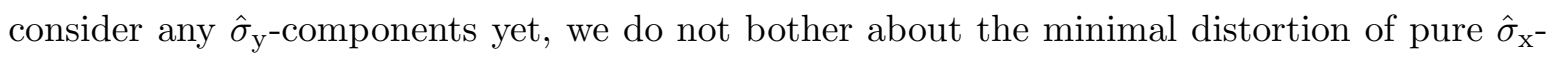
manipulations, which can be estimated to be on the $2^{\text {nd }}$ order of the aberration parameter $\delta \phi_{0}$ that we assumed to be very small anyway. 